\title{
Biochemical markers of muscle damage and high serum concentration of creatine kinase in patients on statin therapy
}

\author{
Adriana AR Nogueira*,1, Célia MC Strunz ${ }^{1}$, Julio Y Takada ${ }^{1}$ \& Antonio P Mansur ${ }^{1}$ \\ ${ }^{1}$ Instituto do Coração (InCor), Hospital das Clínicas HCFMUSP, Faculdade de Medicina, Universidade de São Paulo, SP, Brazil \\ *Author for correspondence: Tel..: +55 112661 5383; Fax: +55 112661 5394; adriana.nogueira@incor.usp.br
}

\begin{abstract}
Aim: Some patients experience statin-associated muscle symptoms (SAMS) and elevated serum concentrations of CK. The relationship between SAMS and biomarkers of muscle damage was examined. Methods: We analyzed 359 consecutive patients taking statins with high CK values. Muscle-related symptoms and biochemical variables, including CK, MB isoenzyme of creatine kinase (CK-MB), troponin and carbonic anhydrase type III were evaluated. Results: SAMS was reported by $181(50.4 \%)$ patients and they had greater BMI $(p=0.021)$ and a trend toward higher CK-MB values $(p=0.064)$. The use of simvastatin (OR: 2.24; 95\% Cl: 1.47-3.42), CK-MB (OR: 1.59; 95\% Cl: 1.02-2.49) and BMI (OR: 1.06; 95\% Cl: 1.01-1.10) were independent variables for SAMS. Conclusion: Simvastatin use, BMI and CK-MB were independent markers of SAMS.
\end{abstract}

First draft submitted: 19 October 2018; Accepted for publication: 22 March 2019; Published online: 3 June 2019

Keywords: carbonic anhydrase III $\bullet$ CK $\bullet$ CK-MB $\bullet$ myalgia $\bullet$ myopathy $\bullet$ myositis $\bullet$ statin

Statins are a class of lipid-lowering drugs that act by inhibiting enzyme HMG-CoA reductase, which catalyzes a limiting step in the cholesterol synthesis process [1]. For this reason, they are widely used in primary and secondary prevention of cardiovascular events [2], with an estimated 25 million users worldwide [3]. Statins are well tolerated; however, some studies have attributed statins to several side effects including but not limited to, muscle pain (myopathy), increased CK, and slightly increased diabetes incidence [4,5]. The muscle symptoms associated with statins may compromise the patient's quality of life and adherence to medication, and consequently, their outcome on cardiovascular events [6].

Statin-related definitions of myopathy are often inconsistent and difficult to interpret. However, the American College of Cardiology (ACC), the American Heart Association (AHA) and the Canadian Working Group (CWG) proposed the following definitions: myopathy - a general term referring to any muscle disease; myalgia - muscle ache or weakness without CK elevation; myositis - muscle symptoms with increased CK levels; rhabdomyolysis muscle symptoms with marked CK elevation (typically substantially greater than ten-times the upper reference limit [URL] and with creatinine elevation) [6,7]. Muscle symptoms that commonly manifest as pain, weakness, stiffness and cramps are more common in physically active patients [8]. However, mild or moderate muscle symptoms are neglected in clinical practice, and consequently their frequency is likely underestimated [9]. A meta-analysis performed with 135 randomized placebo-controlled clinical trials provided evidence of the tolerability and damage of statins. In these studies, statins were associated with an increased risk of diabetes mellitus and elevated hepatic transaminases, although satins were not associated with any significantly detectable effect on myalgia, myopathy and rhabdomyolysis [10]. Conversely, observational studies indicated that $10-25 \%$ of patients treated with statins reported some type of muscular symptom [8,11]. Myopathy occurs most frequently in patients taking high doses of statins. Elderly patients, especially women and those with diabetes with chronic renal failure, appear to be more susceptible to this condition. Drug interaction provides an important risk for the occurrence of myopathies in patients treated with statins [12].

$\mathrm{CK}$ is an enzyme present in tissue and in energy-demanding cells, such as skeletal and cardiac muscles, and is considered the best marker for the detection and monitoring of skeletal muscle diseases [13]. CK-MB, an isoenzyme 
of CK, is found both in cardiac muscle, where it represents approximately $20 \%$ of total CK, and in normal skeletal muscle, where it represents up to $3 \%$ of total CK [14]. Individuals who experience periods of intense exercise, such as marathon runners, may experience a chronic necrotic state of muscle fiber, leading to an increase in CK-MB [15]. In destructive myopathies, such as Duchenne muscular dystrophy, polymyositis and dermatomyositis [16], and in individuals with chronic kidney disease, this enzyme is increased along with total CK [17].

Carbonic anhydrases are metalloproteinases present in all living beings. This enzyme is released into the circulation after muscle injury [18]. Ohta et al. showed that CAIII concentration is increased in the serum of patients with Duchenne muscular dystrophy. CAIII has been shown to be a more sensitive marker than CK in other neurological diseases, such as progressive spinal muscular atrophy and amyotrophic lateral sclerosis [19]. Increased serum CAIII concentration was also found after exercise in healthy individuals along with other markers of muscle damage, such as myoglobin and CK [20].

This study examined the relationships among muscle-related symptoms, lipid profile, biomarkers of statin-related side effects (CK, AST and ALT) and myocardial necrosis (CK-MB, troponin), CRP and CAIII in patients under statin therapy with high serum CK levels.

\section{Methods}

This cross-sectional study analyzed the CK levels of 6692 consecutive outpatients on statins (simvastatin or atorvastatin) from 2009 to 2016 who were older than 18 years of age and undergoing treatment at the Heart Institute (InCor) of the University of São Paulo, São Paulo, Brazil. Of this total, 659 (9.7\%) patients had increased CK enzymatic activity greater than $1 \times$ the URL. The informed consent was obtained from 359 patients, comprising 191 (53\%) women and 168 (47\%) men. The patients underwent an interview in which demographic and clinical characteristics were addressed. The information obtained was confirmed by analyzing the patients' records. The main clinical features analyzed were age, sex, BMI and muscle symptoms presence. Complaints of muscle pain reported by patients and considered relevant and associated with statin use were lower limb pain, stiffness, cramps and muscle weakness. These symptoms could be classified as myositis according to the definitions proposed by the American College of Cardiology (ACC), the American Heart Association (AHA) and the Canadian Working Group (CWG). However, since there was no histopathological evidence of muscle impairment, we classified muscular symptoms as statin-associated muscle symptoms (SAMS) [6,7]. Laboratory tests included analysis of serum concentrations of triglycerides, total cholesterol, HDL cholesterol, VLDL cholesterol, LDL cholesterol, biomarkers of statin-related side effects (CK, AST and ALT), myocardial necrosis (CK-MB, troponin), CRP and CAIII. Exclusion criteria were chronic renal insufficiency (creatinine $>2.0 \mathrm{mg} / \mathrm{dl}$ ), a history of hypothyroidism or treatment with a thyroid hormone, a history of inflammatory and/or infectious disease, a history of skeletal muscle diseases, previous diagnosis of cancer or therapy with a fibrate. Patients who reported exercising up to three days before blood collection were also excluded.

This study was approved by the Ethics Committee of the University of São Paulo Medical School Hospital (CAPPesq: 1119/09).

\section{Laboratory measurements}

CK and liver enzyme (AST and ALT) tests were performed by the automated kinetic method. Total cholesterol, highdensity lipoprotein cholesterol, triglycerides, and creatinine tests were performed by the enzymatic colorimetric method. Glucose and urea were evaluated by the automated enzymatic method. The assays were performed using Dimension RXL equipment, Siemens Healthcare (NJ, USA), with the dedicated reagents. hsCRP was quantified by immunonephelometry using automated BN II Systems equipment with a specific kit (Siemens Healthcare Diagnostics Products, Marburg, Germany). CK-MB and troponin I were measured by chemiluminescent immunoassays performed using an Advia Centaur system (Siemens Healthcare Diagnostic Products) with specific kits. CAIII was determined by enzyme-immunoassay using a commercial Cloud-Clone Corp kit (TX, USA).

\section{Statistical analysis}

All variables were analyzed descriptively. For the continuous variables, data were expressed as the mean \pm standard deviation (SD). Absolute and relative frequencies were calculated for the categorical variables. The Pearson's test was used to measure the statistical association between two variables. Student's t-test was performed for variables with a normal distribution, which was verified by analysis of the equality of variances (Folded F). Depending on the result of this analysis, the Pooled method (variances with $\mathrm{p} \geq 0.05$ ) or the Satterthwaite method (variances with 
Table 1. Clinical and laboratory variables related to creatine kinase levels.

\begin{tabular}{|c|c|c|c|c|c|c|c|}
\hline Clinical and laboratory variables & $\mathrm{n}$ (total) & Total & $\mathrm{n}$ & CK (1-3 x URL) & $\mathbf{n}$ & CK ( $>3 \times$ URL $)$ & p-value \\
\hline Age, years & 359 & $61.6 \pm 10.1$ & 339 & $61.7 \pm 10.3$ & 20 & $59.7 \pm 7.2$ & 0.383 \\
\hline $\operatorname{Sex}(\%)$ & 359 & $\begin{array}{l}168 \text { men (46.8) } \\
191 \text { women (53.2) }\end{array}$ & 339 & $\begin{array}{l}158 \text { men (46.6) } \\
181 \text { women }(53.4)\end{array}$ & 20 & $\begin{array}{l}10 \text { men }(50) \\
10 \text { women }(50)\end{array}$ & 0.768 \\
\hline BMI $\left(\mathrm{kg} / \mathrm{m}^{2}\right)$ & 359 & $28.9 \pm 5.4$ & 339 & $28.8 \pm 4.9$ & 20 & $30.1 \pm 10.3$ & 0.566 \\
\hline SAMS $(\%)$ & 181 & 50.4 & 169 & 50.0 & 12 & 60.0 & 0.378 \\
\hline Diabetes (\%) & 159 & 44.3 & 149 & 44.0 & 10 & 50.0 & 0.605 \\
\hline Simvastatin (mg/day) & 171 & $24.8 \pm 13.7$ & 162 & $25.0 \pm 13.8$ & 9 & $21.1 \pm 11.7$ & 0.408 \\
\hline Atorvastatin (mg/day) & 188 & $34.5 \pm 20.6$ & 177 & $34.5 \pm 20.6$ & 11 & $34.5 \pm 20.2$ & 0.997 \\
\hline CK $(U / I)$ & 359 & $407.0 \pm 405.6$ & 339 & $346.8 \pm 125.4$ & 20 & $1427.3 \pm 1288.0$ & $0.001^{\dagger}$ \\
\hline CK-MB (ng/ml) & 359 & $4.1 \pm 3.6$ & 339 & $3.7 \pm 2.5$ & 20 & $10.6 \pm 9.3$ & $0.004^{\dagger}$ \\
\hline Carbonic anhydrase III (pg/ml) & 231 & $208.2 \pm 89.6$ & 217 & $209.0 \pm 90.5$ & 14 & $196 \pm 76.8$ & 0.601 \\
\hline Total cholesterol (mg/dl) & 355 & $181.2 \pm 50.2$ & 335 & $180.6 \pm 49.9$ & 20 & $191.7 \pm 55.4$ & 0.337 \\
\hline HDL cholesterol (mg/dl) & 355 & $45.9 \pm 15.1$ & 335 & $45.9 \pm 14.7$ & 20 & $46.6 \pm 20.9$ & 0.889 \\
\hline LDL cholesterol (mg/dl) & 354 & $107.1 \pm 42.6$ & 334 & $106.5 \pm 42.5$ & 20 & $117.8 \pm 43.8$ & 0.249 \\
\hline Triglycerides (mg/dl) & 355 & $148.9 \pm 174.6$ & 335 & $149.2 \pm 178.9$ & 20 & $143.3 \pm 76.7$ & 0.767 \\
\hline Glucose (mg/dl) & 341 & $121.0 \pm 50.0$ & 322 & $121.7 \pm 51.0$ & 19 & $110.0 \pm 28.5$ & 0.113 \\
\hline Urea (mg/dl) & 330 & $41.7 \pm 15.8$ & 310 & $41.5 \pm 14.6$ & 20 & $45.9 \pm 29.4$ & 0.506 \\
\hline Creatinine $(\mathrm{mg} / \mathrm{dl})$ & 331 & $1.1 \pm 0.3$ & 311 & $1.10 \pm 0.29$ & 20 & $1.16 \pm 0.41$ & 0.545 \\
\hline AST $(U / I)$ & 308 & $32.9 \pm 14.4$ & 290 & $31.5 \pm 12.5$ & 18 & $54.8 \pm 23.1$ & $0.001^{\dagger}$ \\
\hline $\operatorname{ALT}(U / I)$ & 317 & $45.6 \pm 20.5$ & 298 & $44.7 \pm 20.2$ & 19 & $58.6 \pm 21.8$ & $0.004^{\dagger}$ \\
\hline $\mathrm{hsCRP}(\mathrm{mg} / \mathrm{dl})$ & 349 & $3.9 \pm 5.5$ & 329 & $3.92 \pm 5.43$ & 20 & $4.4 \pm 6.3$ & 0.700 \\
\hline Troponin I (ng/l) & 359 & $0.01 \pm 0.01$ & 339 & $0.01 \pm 0.01$ & 20 & $0.02 \pm 0.02$ & 0.526 \\
\hline \multicolumn{8}{|c|}{$\begin{array}{l}\text { Values expressed as the mean ( } \pm \text { standard deviation). } \\
\text { CK } 1-3 \times \text { URL - male: } 309-924 \text { U/l; female: 193-476 U/l; CK >3 } \times \text { URL - male: }>925 \text { U/l; female: }>477 \text { U/l. } \\
\text { †The significance level adopted was } 5 \%(p \leq 0.05) \text {. } \\
\text { BMI: Body mass index; SAMS: Statin-associated muscle symptom; URL: Upper reference limit. }\end{array}$} \\
\hline
\end{tabular}

$\mathrm{p}<0.05)$ was used. The $\chi^{2}$ was performed for categorical variables. Multivariate logistic regression analysis was performed using a stepwise approach, considering SAMS as the dependent variable. The independent variables to be tested in the model were selected from those considered risk factors for SAMS, which included age, sex, BMI, medication and the biochemical markers CK, CK-MB, AST and CAIII. Odds ratios (ORs) were calculated as part of the logistic regression analysis. Statistical analyses were performed using the SAS program, version 9.3 (SAS Institute Inc., NC, USA).

\section{Results}

CK results were evaluated in 6692 outpatients who used some type of statin. Of these patients, 6041 (90.3\%) had CK values up to $1 \times$ the URL, $109 \pm 48.8 \mathrm{U} / 1$, and 651 (9.7\%) had values greater than $1 \times \mathrm{URL}, 420.5 \pm 427 \mathrm{U} / 1$, considering the reference range of 26-192 U/1 for females and 39-308 U/1 for males. Of the total number of patients with altered CK, 359 formally consented to participate in the study. Of these patients, 46.8\% $(n=168)$ were men. The BMI of these patients was $28.9 \pm 5.4 \mathrm{~kg} / \mathrm{m}^{2}$. Regarding the statin type, $47.6 \%(\mathrm{n}=171)$ of the patients used simvastatin at a mean daily dose of $24.8 \pm 13.7 \mathrm{mg}$, and $52.4 \%(\mathrm{n}=188)$ used atorvastatin, with a mean dose of $34.5 \pm 20.6 \mathrm{mg}$ daily.

CK values were categorized as greater than $1-3 \times$ the URL $(n=339)$ and greater than $3 \times$ the URL $(n=20)$, which corresponded to CK values of $346.8 \pm 125.4 \mathrm{U} / \mathrm{l}(1-3 \times \mathrm{URL})$ and $1427.3 \pm 1288.0 \mathrm{U} / \mathrm{l}(>3 \times \mathrm{URL} ; \mathrm{p}=0.001)$, respectively. CK values had positive correlations with BMI $(r=0.25 ; \mathrm{p}<0.001)$, creatinine $(\mathrm{r}=0.16 ; \mathrm{p}=0.004)$, AST $(r=0.50 ; p<0.001)$, ALT $(r=0.23 ; p<0.001)$ and CK-MB $(r=0.59 ; p<0.001)$. The clinical and laboratory variables for CK categorized groups are shown in Table 1. SAMS symptoms were reported by 180 patients (50.1\%). The clinical and laboratory variables of patients with and without SAMS are shown in Table 2. Regarding the statin type, 103 patients with SAMS (57.5\%) used simvastatin $(26.1 \pm 14.5 \mathrm{mg} /$ day), whereas 77 patients $(42.5 \%)$ used atorvastatin $(33.6 \pm 21.8 \mathrm{mg} /$ day; $\mathrm{p}=0.0002)$. The multivariate logistic regression analysis using SAMS as the dependent variable was adjusted for the variables of age, sex, BMI, medication, CK and CK-MB. The 


\begin{tabular}{|c|c|c|c|c|c|}
\hline Clinical and laboratory variables & $\mathrm{n}$ & Absence of SAMS & $n$ & Presence of SAMS & p-value \\
\hline Age, years & 178 & $61.9 \pm 10.6$ & 181 & $61.4 \pm 9.6$ & 0.624 \\
\hline $\operatorname{Sex}(\%)$ & 178 & $\begin{array}{l}86 \text { men }(48.3) \\
92 \text { women (51.7) }\end{array}$ & 181 & $\begin{array}{l}82 \text { men }(45.3) \\
99 \text { women }(54.7)\end{array}$ & 0.568 \\
\hline BMI $\left(\mathrm{kg} / \mathrm{m}^{2}\right)$ & 178 & $28.2 \pm 5.1$ & 181 & $29.5 \pm 5.6$ & $0.021^{\dagger}$ \\
\hline Diabetes (\%) & 80 & 44.9 & 79 & 44.0 & 0.841 \\
\hline Simvastatin (mg/day) & 68 & $22.8 \pm 12.2$ & 103 & $26.1 \pm 14.5$ & 0.133 \\
\hline Atorvastatin (mg/day) & 111 & $35.1 \pm 19.8$ & 77 & $33.6 \pm 21.8$ & 0.624 \\
\hline CK (U/l) & 178 & $387.8 \pm 228.1$ & 181 & $426.0 \pm 524.6$ & 0.370 \\
\hline CK-MB (ng/ml) & 178 & $3.7 \pm 3.2$ & 181 & $4.4 \pm 4.0$ & 0.064 \\
\hline Carbonic anhydrase III (pg/ml) & 131 & $211.3 \pm 93.4$ & 100 & $204.0 \pm 84.6$ & 0.549 \\
\hline Total cholesterol (mg/dl) & 177 & $182 \pm 54.7$ & 178 & $180.4 \pm 45.5$ & 0.762 \\
\hline HDL cholesterol (mg/dl) & 177 & $46.6 \pm 14.8$ & 178 & $45.3 \pm 15.3$ & 0.419 \\
\hline LDL cholesterol (mg/dl) & 177 & $109.0 \pm 45.8$ & 177 & $105.2 \pm 39.2$ & 0.394 \\
\hline Triglycerides (mg/dl) & 177 & $145.5 \pm 230.4$ & 178 & $152.2 \pm 90.6$ & 0.716 \\
\hline Glucose (mg/dl) & 174 & $124.3 \pm 57.3$ & 167 & $117.6 \pm 41.1$ & 0.213 \\
\hline Urea (mg/dl) & 172 & $42.1 \pm 17.3$ & 158 & $41.3 \pm 14.1$ & 0.667 \\
\hline Creatinine $(\mathrm{mg} / \mathrm{dl})$ & 173 & $1.13 \pm 0.30$ & 158 & $1.07 \pm 0.28$ & 0.066 \\
\hline AST (U/I) & 155 & $32.6 \pm 13.4$ & 153 & $33.2 \pm 15.3$ & 0.676 \\
\hline $\operatorname{ALT}(\mathrm{U} / \mathrm{I})$ & 161 & $44.3 \pm 21.3$ & 156 & $46.8 \pm 19.7$ & 0.277 \\
\hline hsCRP (mg/dl) & 176 & $4.0 \pm 6.2$ & 173 & $3.9 \pm 4.7$ & 0.741 \\
\hline Troponin I (ng/l) & 177 & $0.01 \pm 0.02$ & 180 & $0.01 \pm 0.02$ & 0.664 \\
\hline
\end{tabular}

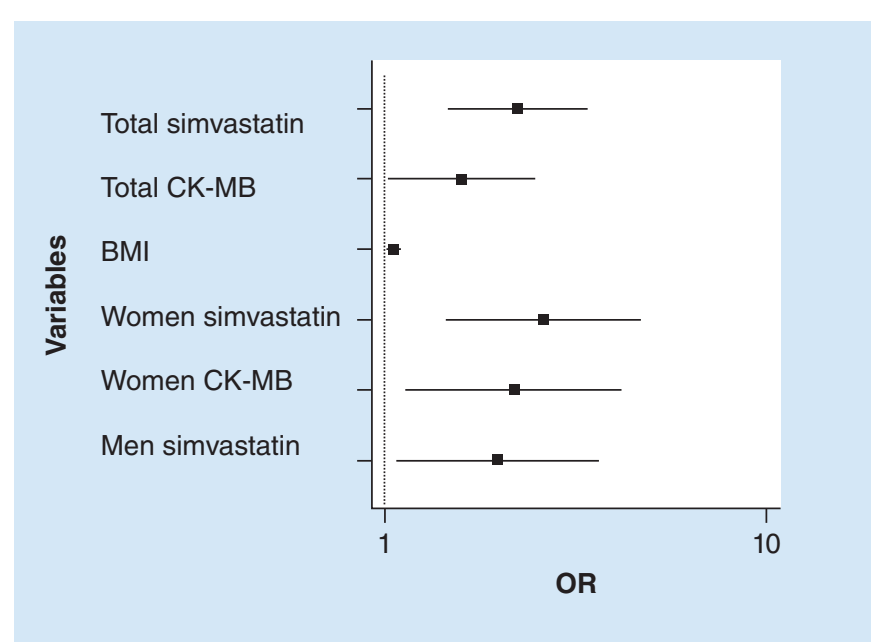

Figure 1. Logistic regression analysis for dependent variable myositis and independent; age, sex, BMI, medication

(simvastatin/atorvastatin), aspartate aminotransferase, CK and MB isoenzyme of creatine kinase, for the total population, women and men.

BMI: Body mass index; OR: Odds ratio.

independent variables for SAMS in the total population were medication (simvastatin/atorvastatin; OR: 2.24; 95\% CI: 1.47-3.42), CK-MB (OR: 1.59; 95\% CI: 1.02-2.49) and BMI (OR: 1.06; 95\% CI: 1.01-1.10). When the dependent variable was the presence of SAMS in women adjusted for the same variables, the independent variables were the use of simvastatin (OR: 2.61; 95\% CI: 1.44-4.71) and CK-MB (OR: 2.18; 95\% CI: 1.14-4.18). When the dependent variable was the presence of SAMS in men adjusted for the same variables, the dependent variable was the use of simvastatin (OR: 1.97; 95\% CI: 1.07-3.65). The inclusion of carbonic anhydrase in the model did not change the final results of the logistic regressions above. These results are represented in Figure 1. CAIII was analyzed in 231 patients and resulted in a mean value of $208.2 \mathrm{pg} / \mathrm{ml} \pm 89.6 \mathrm{pg} / \mathrm{ml}$. CAIII had positive correlations with total cholesterol $(r=0.23 ; p=0.001)$, low-density lipoprotein cholesterol $(r=0.18 ; p=0.005)$, triglycerides 
$(\mathrm{r}=0.18 ; \mathrm{p}=0.007)$ and a negative correlation with CK-MB $(\mathrm{r}=0.19 ; \mathrm{p}=0.004)$. Patients with and without SAMS did not have different concentrations of CAIII $(211.3 \pm 93.4 \mathrm{pg} / \mathrm{ml}$ vs $204.0 \pm 84.6 \mathrm{pg} / \mathrm{ml} ; \mathrm{p}=0.549)$. The CAIII values were similar in patients with CK values of $1-3 \times$ the URL and CK values $>3 \times$ the URL. On the other hand, patients with normal CK-MB values had higher concentrations of CAIII than patients with increased CK-MB (220.7 $\pm 90.4 \mathrm{pg} / \mathrm{ml}$ vs $191.5 \pm 86.2 \mathrm{pg} / \mathrm{ml} ; \mathrm{p}=0.014)$.

\section{Discussion}

This study showed simvastatin use, BMI and high serum concentrations of CK-MB as independent markers of SAMS. In our study, approximately $10 \%$ of 6692 patients on statin therapy had CK values greater than $1 \times$ the URL and half of the 359 patients analyzed reported the presence of some muscular symptom (weakness, muscular pain, stiffness or cramps). This result was much higher than that found in the literature, although studies reported different cut-off values for elevated serum CK [21]. This study took into account any elevation of CK in patients on statin therapy, regardless of the presence of muscle symptoms. Few studies have reported the frequency of CK abnormalities below $10 \times$ the URL [22]. Our results differed from those found by Smith et al., where only $0.3 \%$ of the 1040 patients monitored had significantly elevated CK attributed to statin therapy [23]. They defined significant elevations of CK as more than $5 \times$ the URL, while in our study, only $0.06 \%$ of 6692 the patients met this cut-off value for elevated CK. El-Salem et al. reported an even higher incidence of myositis of $21 \%$ of 345 patients treated with statins, but only two patients with myositis had an elevated CK level $3 \times$ the URL [24]. Similar to our study, Fung and Crook's study in a lipid clinic observed that 108 (10.2\%) of outpatients on statin therapy experienced muscle impairment and were associated with higher levels of serum CK [25]. Both studies considered any elevation of CK, and very few patients had elevated CK levels greater $3 \times$ the URL. Serum CK elevation is considered the best marker of skeletal muscle damage, but the relationship between elevation of CK concentration and SAMS is controversial. In our study, we also did not observe any relationship between these variables, making CK a nonspecific biomarker of SAMS presence.

In the present study, a high number of patients had elevated CK-MB values (36.2\%). Although patients with SAMS showed higher CK-MB values than asymptomatic patients, the difference was not significant, probably because many patients with SAMS had total CK values just above the URL. On the other hand, patients with CK values $>3 \times$ the URL had a greater increase in CK-MB mass than those with CK values $1-3 \times$ the URL. Siegel et al. have shown that the concentration of CK-MB ranges from 1 to $7 \%$ of the total CK per grams of total protein in the skeletal muscle [15]. In the literature, few studies have compared the elevation of CK-MB with the presence of myositis or other types of muscular symptoms and treatment with statins. However, some studies have reported the elevation of this enzyme in the presence of chronic muscular diseases, such as dermatomyositis and polymyositis [26].

Nakahara et al. had already observed that alterations of cell membranes promoted by statins could result in CK leakage before the onset of muscular symptoms [27].

Also the increase in CK-MB level in the absence of myocardial damage may be related to a regenerative response of muscle tissue to the cellular trauma produced by the use of statins. Siegel et al. observed in marathon runners, a balance between injury and repair in skeletal muscle and in this case the CK-MB activity may be considered a biomarker [15].

High serum concentration of CK-MB could be a marker of myositis in outpatients under statin therapy with elevation of CK activity. In our study, the CK-MB value had a significant positive correlation with the CK value and was an independent predictor of the presence of SAMS in the multivariate regression.

A high serum concentration of CK-MB but not CK could be a marker of myositis in outpatients under statin therapy with greater elevation of CK levels. In our study, the CK-MB value had a significant positive correlation with the CK value and was an independent predictor of the presence of SAMS in the multivariate regression.

On the other hand, care should be taken in interpreting CK-MB levels in the emergency department of patients on statin therapy. CK-MB may be a confounding variable in these patients for the differential diagnosis of an acute coronary syndrome in facilities where CK-MB is still used as a diagnostic marker of myocardial necrosis.

In this study, AST and ALT were found to be greater than $1 \times$ the URL in 21.7 and $11.3 \%$ of patients, respectively. Simvastatin and atorvastatin showed the same effects on elevating transaminases, which was likely due to the use of low to moderate doses of statins. These results were similar to those of Clark et al., who did not show any differences among lower to moderate doses of atorvastatin and simvastatin on moderate to severe hepatotoxicity. High hepatic transaminases were only observed when they compared high-dose atorvastatin to low-dose simvastatin [28]. 
Hepatic adverse effects are one of the common side effects reported with statins. Our results showed a significant difference observed in ALT values in CK $(1-3 \times$ URL) and CK $(>3 \times$ URL) groups. But ALT maximum values are only slightly above the normal range and could not be associated to hepatic injury.

On the other hand, high levels of AST obtained in CK $(>3 \times$ URL) group were expected since this enzyme is present in skeletal muscle.

CAIII was not significantly different between patients with CK values of $1-3 \times$ the URL and those with values $>3 \times$ the URL. The CAIII values also did not change significantly in patients with muscle complaints, although they all had altered levels of CK. One hypothesis explaining this finding is based on the possible inhibitory properties of statins in relation to carbonic anhydrases. $\beta$-hydroxybutyric acid (BHB) is a ketone body produced during the ketogenic diet. In humans, $\mathrm{BHB}$ is synthesized in the liver from acetyl-coA and inhibits various isoforms of carbonic anhydrases. This fragment is present in the molecules of most statins. For this reason, statins have a submicromolar inhibition pattern of the anhydrases [29].

BMI is also an independent variable for SAMS. Patients with SAMS had a higher BMI than those who did not report muscle pain, which is different from reports in the literature where low BMI is considered a risk factor for the occurrence of muscular symptoms related to the use of statins [10].

SAMS occurred more frequently in patients using simvastatin (57.5\%), with a mean daily dose of $26.1 \pm 14.5 \mathrm{mg}$, in spite of atorvastatin being given at higher dosages $(33.6 \pm 21.8 \mathrm{mg})$. According to the multivariate analysis, the use of simvastatin was an independent predictor of the occurrence of SAMS in the study population. There are no clinical trials directly comparing the risk of myotoxicity and equivalent doses of individual statins. A review study suggested that high doses $(80 \mathrm{mg})$ but not low $(20 \mathrm{mg})$ to moderate $(40 \mathrm{mg})$ doses of simvastatin were associated with a higher incidence of myotoxicity when compared with the maximum doses approved for atorvastatin [30]. However, a recent meta-analysis study did not show a higher incidence of myositis for any dose of simvastatin and atorvastatin [10]. Another possible explanation to justify the association between myositis and the use of simvastatin is drug interaction. Despite the exclusion of clinically significant diseases, these individuals may be taking drugs such as clopidogrel, fluoxetine, verapamil, diltiazem and amiodarone, which inhibit cytochrome $\mathrm{p} 450$, resulting in higher serum concentrations of simvastatin. In the of STOMP study, healthy randomized patients were assigned to treatment with $80 \mathrm{mg}$ atorvastatin or placebo. According to the definition of myalgia used in the study, $9.4 \%$ of statin users developed muscular symptoms versus $4.6 \%$ of patients treated with placebo $(p=0.05)$ [31]. In the PRIMO study the statin type was also considered an independent predictor of the appearance of muscular symptoms. Treatment with high doses of simvastatin ( $40 \mathrm{or} 80 \mathrm{mg} /$ day) and atorvastatin ( $40 \mathrm{or} 80 \mathrm{mg} /$ day) was associated with an increase in the occurrence of these symptoms ( $p<0.0001$ and $p=0.035$, respectively) [8].

The present cross-sectional study has some limitations. There was only one measurement per patient for all laboratory data and no previous CK value at study entry. Time frame of statin therapy before patient inclusion in the study was unknown and the muscle pain was self-reported, which may have introduced some bias in the results. Also only patients with cardiovascular disease were studied.

In conclusion, higher BMI, higher serum concentration of CK-MB and simvastatin use but not CK and CAIII were independently associated with SAMS. CK-MB could be a biomarker of skeletal muscle damage intensity in subjects under statins with a high serum concentration of CK.

\section{Financial \& competing interests disclosure}

The authors have no relevant affiliations or financial involvement with any organization or entity with a financial interest in or financial conflict with the subject matter or materials discussed in the manuscript. This includes employment, consultancies, honoraria, stock ownership or options, expert testimony, grants or patents received or pending, or royalties.

No writing assistance was utilized in the production of this manuscript.

Ethical conduct of research

This study was approved by the Ethics Committee of the University of São Paulo Medical School Hospital (CAPPesq: 1119/09). In addition, for investigations involving human subjects, informed consent has been obtained from the participants involved. 
Summary points

Statin-associated muscle symptoms definitions

- Myopathy is generally defined as any disease of muscle.

- Myalgia is defined as muscle aches or weakness in the absence of creatine kinase (CK) elevation.

- Myositis is defined as elevated CK in the presence of muscle symptoms.

- Rhabdomyolysis is defined as pronounced CK elevation ( $>10 \times$ normal limit), with serum creatinine elevation, in the presence of muscle symptoms.

Biological basis of statin-induced myopathy

- There are some hypotheses that seek to explain the mechanism by which statins induce muscular toxicity.

- One is that statin could lower cholesterol content in the sarcolemma, resulting in changes in membrane fluidity, altering its integrity and leading to degradation and necrosis.

- Another proposed mechanism of statin-induced myopathy is a deficiency of coenzyme Q10 which is a metabolite of the 3-hydroxy-3-methylglutaryl-coenzyme A reductase pathway. Statins inhibit the synthesis of mevalonate, a precursor of coenzyme Q10 that plays a key role in the electron transport chain and a reduction of this coenzyme may result in abnormal respiratory function of the mitochondria that may lead to myopathy.

Total CK \& MB isoenzyme of CK

- Total CK has two subunits, M and B, which make up the three main groups of isoenzymes CK-MM, CK-MB and CK-BB. CK-MM is present in skeletal and cardiac musculature and accounts for about 97 and $80 \%$ of total CK activity, respectively. CK-BB is found primarily in the brain. CK-MB is predominantly found in the cardiac muscle, where it makes up about $20 \%$ of total CK activity. Despite the wide use of CK-MB as a specific marker in the detection of myocardial damage, this isoenzyme is also present in the skeletal muscle $(0-3 \%)$. Severe exercise situations and destructive myopathies, such as Duchenne muscular dystrophy, inflammatory muscle disease, polymyositis and dermatomyositis, CK-MB are increased together with total CK.

Carbonic anhydrases

- CAIII is a $28 \mathrm{kDa}$ cytosolic isoenzyme located almost exclusively in skeletal muscle, where it is the major soluble protein and is possibly involved in the diffusion of $\mathrm{CO}_{2}$ into the tissue. This enzyme is released into the circulation after muscle injury.

Future perspective

- A biomarker of muscle symptoms experienced by patients under statin therapy would be important for the diagnosis of the myopathy. Also it would allow choosing the best therapy for each patient taking into account the cholesterol effect and the muscle-related symptoms.

\section{Open access}

This work is licensed under the Attribution-NonCommercial-NoDerivatives 4.0 Unported License. To view a copy of this license, visit http://creativecommons.org/licenses/by-nc-nd/4.0/

\section{References}

Papers of special note have been highlighted as: • of interest; $\bullet \bullet$ of considerable interest

1. Grundy SM. HMG-CoA reductase inhibitors for treatment of hypercholesterolemia. N. Engl. J. Med. 319, 24-33 (1988).

2. Law M, Rudnicka AR. Statin safety: a systematic review. Am. J. Cardiol. 97(8A), 52C-60C (2006).

3. Mitka M. Expanding statin use to help more at-risk patients is causing financial heartburn. JAMA 290(17, 2243-2245 (2003).

4. Mancini GB, Baker S, Bergeron J et al. Diagnosis, prevention, and management of statin adverse effects and intolerance: Canadian Consensus Working Group Update (2016). Can. J. Cardiol. 32(Suppl. 7), S35-S65 (2016).

5. Richardson K, Schen M, French B et al. Statin and cognitive function: a systematic review. Ann. Intern. Med. 159, 688-697 (2013).

6. Pasternak RC, Smith SC Jr, Bairey-Merz CN, Grundy SM, Cleeman JI, Lenfant C. ACC/AHA/NHLBI Clinical advisory on the use and safety of statins. Stroke 33(9), 2337-2341 (2002).

-. Definitions of myopathy, myalgia, myositis and rhabdomyolysis.

7. Mancini GB, Tashakkor AY, Baker $S$ et al. Diagnosis, prevention, and management of statin adverse effects and intolerance: Canadian Working Group Consensus update. Can. J. Cardiol. 29(12), 1553-1568 (2013).

8. Bruckert E, Hauyem G, Dejager S, Yau C, Begand B. Mild to moderate muscular symptoms with high-dosage statin therapy in hyperlipidemic patients - the PRIMO study. Cardiovasc. Drugs Ther. 19(6), 403-414 (2005).

-• Observational study on muscle symptoms associated with statin therapy.

9. Sinzinger H, Wolfram R, Peskar BA. Muscular side effects of statins. J. Cardiovasc. Pharmacol. 40(2), 163-171 (2002).

10. Naci H, Brugts J, Ades T. Comparative tolerability and harms of individual statins: a study-level network meta-analysis of 246955 participants from 135 randomized, controlled trials. Circ. Cardiovasc. Qual. Outcomes 6(1), 390-399 (2013). 
- Meta-analysis of tolerability of statins and adverse effects.

11. Cohen JD, Brinton EA, Ito MK, Jacobson TA. Understanding statin use in America and gaps in patient education (USAGE): an internet-based survey of 10.138 current and former statin users. J. Clin. Lipidol. 6(3), 208-215 (2012).

12. Mancini GB, Baker S, Bergeron J et al. Diagnosis, prevention, and management of statin adverse effects and intolerance: proceedings of a Canadian Working Group Consensus Conference. Can. J. Cardiol. 27(5), 635-662 (2011).

13. Wu AH, Perryman MB. Clinical applications of muscle enzymes and proteins. Curr. Opin. Rheumatol. 4(6), 815-820 (1992).

14. Christenson RH, Azzazy HM. Biochemical markers of the acute coronary syndromes. Clin. Chem. 44(8), 1855-1864 (1998).

15. Siegel AJ, Silverman LM, Evans WJ. Elevates skeletal muscle creatine kinase MB isoenzyme levels in marathon runners. JAMA 250(20), 2835-2837 (1983).

- Increased MB isoenzyme of creatine kinasein healthy individuals.

16. Kiely PD, Bruckner FE, Nisbet JA, Daghir A. Serum skeletal troponin I in a inflammatory muscle disease: relation to creatine kinase, CK-MB and cardiac troponin I. Ann. Rheum. Dis. 59(9), 750-751 (2000).

17. Ricchiuti V, Voss EM, Ney A, Odland M, Apple FS. Skeletal muscle expression of creatine kinase-B in end-stage renal disease. Clin. Proteomics 1(1), 33-39 (2004).

18. Supuran CT. Structure-based drug discovery of carbonic anhydrase inhibitors. J. Enzyme Inhib. Med. Chem. 27(6), 759-772 (2012).

19. Ohta M, Itagaki Y, Itoh N, Hayashi K, Nishitani H, Ohta K. Carbonic anhydrase III in serum in muscular dystrophy and other neurological disorders: relationship with creatine kinase. Clin. Chem. 37(1), 36-39 (1991).

- Relationship between total creatine kinase and carbonic anhydrase III.

20. Väänänen HK, Leppilampi M, Vuori J, Takala TE. Liberation of muscle carbonic anhydrase into serum during extensive exercise. J. Appl. Physiol. 61(2), 561-564 (1986).

21. Millán J, Pedro-Botet J, Climent E, Millán J, Rius J. Statin associated myopathy in clinical practice. Results of DAMA study. Clin. Investig. Arterioscler. 29(1), 7-12 (2017).

22. Thompson PD, Clarkson P, Karas RH. Statin-associated myopathy. JAMA 289(13), 1681-1690 (2003).

23. Smith CC, Bernstein LI, Davis RB, Rind DM, Shmerling RH. Screening for statin-related toxicity: the yield of transaminase and creatine kinase measurements in a primary care setting. Arch. Intern. Med. 163(6), 688-692 (2003).

24. El-Salem K, Ababneh B, Rudnicki S et al. Prevalence and risk factors of muscle complications secondary to statins. Muscle Nerve 44(6), 877-878 (2011).

25. Fung EC, Crook MA. Statin myopathy: a lipid clinic experience on the tolerability of statin rechallenge. Cardiovasc. Ther. 30(5), e212-e218 (2012).

26. Erlacher $\mathrm{P}$, Lercher A, Falkensammer $\mathrm{J}$ et al. Cardiac troponin and beta-type myosin heavy chain concentrations in patients with polymyositis or dermatomyositis. Clin. Chim. Acta 306(1-2), 27-33 (2001).

27. Nakahara K, Kuriyama M, Yoshidome H et al. Experimental simvastatin-induced myopathy in rabbits. J. Neurol. Sci. 113(1), 114-117 (1992).

28. Clarke AT, Johnson PC, Hall GC, Ford I, Mills PR. High dose atorvastatin associated with increased risk of significant hepatotoxicity in comparison to simvastatin in UK GPRD cohort. PLoS ONE 11(3), e0151587 (2016).

29. Parkkila S, Vullo D, Maresca A, Carta F, Scozzafava A, Supuran CT. Serendipitous fragment-based drug discovery: ketogenic diet metabolites and statins effectively inhibit several carbonic anhydrases. Chem. Commun. 48(29), 3551-3553 (2012).

-. Hypothesis of possible inhibitory properties of statins in relation to carbonic anhydrases.

30. Backes JM, Howard PA, Ruisinger JF, Moriarty PM. Does simvastatin cause more myotoxicity compared with other statins? Ann Pharmacother. 43(12), 2012-2020 (2009).

31. Parker BA, Capizzi JA, Grimaldi AS et al. Effect of statins on skeletal muscle function. Circulation 127(1), 96-103 (2013). 